\title{
Insights into mRNP biogenesis provided by new genetic interactions among export and transcription factors
}

\author{
Francisco Estruch ${ }^{1 *}$, Christine Hodge ${ }^{2}$, Natalia Gómez-Navarro ${ }^{1}$, Lorena Peiró-Chova ${ }^{1}$, Catherine V Heath ${ }^{2}$ \\ and Charles N Cole $^{2}$
}

\begin{abstract}
Background: The various steps of mRNP biogenesis (transcription, processing and export) are interconnected. It has been shown that the transcription machinery plays a pivotal role in mRNP assembly, since several mRNA export factors are recruited during transcription and physically interact with components of the transcription machinery. Although the shuttling DEAD-box protein Dbp5p is concentrated on the cytoplasmic fibrils of the NPC, previous studies demonstrated that it interacts physically and genetically with factors involved in transcription initiation.

Results: We investigated the effect of mutations affecting various components of the transcription initiation apparatus on the phenotypes of mRNA export mutant strains. Our results show that growth and mRNA export defects of $d b p 5$ and mex67 mutant strains can be suppressed by mutation of specific transcription initiation components, but suppression was not observed for mutants acting in the very first steps of the pre-initiation complex (PIC) formation.

Conclusions: Our results indicate that mere reduction in the amount of mRNP produced is not sufficient to suppress the defects caused by a defective mRNA export factor. Suppression occurs only with mutants affecting events within a narrow window of the mRNP biogenesis process. We propose that reducing the speed with which transcription converts from initiation and promoter clearance to elongation may have a positive effect on mRNP formation by permitting more effective recruitment of partially-functional mRNP proteins to the nascent mRNP.
\end{abstract}

Keywords: mRNA export, Transcription, Dbp5p, Mex67p, Nuclear Pore Complex

\section{Background}

In eukaryotic cells, synthesis of RNA and protein occur in separate cellular compartments, necessitating the continuous transport of macromolecules between the nucleus and cytoplasm. The transport of both RNAs and proteins takes place through the nuclear pore complexes (NPCs) in an energy-dependent process mediated by saturable transport receptors (for reviews, see [1,2]). During mRNA biogenesis, mRNAs are packaged into ribonucleoprotein complexes (messenger ribonucleoprotein particles, mRNPs). Many of the protein components of mRNPs are removed from mRNPs as they are translocate

\footnotetext{
* Correspondence: estruch@uv.es

${ }^{1}$ Departamento de Bioquímica y Biología Molecular, Universitat de Valencia, Burjassot, Spain

Full list of author information is available at the end of the article
}

through NPCs. The essential mRNA export factor Dbp5p/Rat8p, a member of the DEAD-box family of proteins [3] binds to the cytoplasmic filaments of the NPC, where its ATPase is activated by Gle1p triggering removal of mRNP proteins $[4,5]$. There is evidence that the mRNA export receptor Mex67p and the RNAbinding protein Nab2p, both of which accompany the mRNP through NPCs, are removed from mRNPs by Dbp5p acting at the cytoplasmic filaments $[6,7]$. However, Dbp5p is not restricted to the NPC. It shuttles between nucleus and cytoplasm and can be detected throughout the cell $[8,9]$. Its homologue in Chironomus titans is recruited to mRNA during transcription [10]. Consistent with this, we identified both genetic and physical interactions between Dbp5p and factors involved in transcription initiation, including TFIIH and

\section{Biomed Central}


the C-terminal domain (CTD) of RNA polymerase II (RNA pol II) [11].

The various steps of mRNP biogenesis are coordinated and this is reflected in the extensive interactions that occur between RNA pol II, other components of the transcription machinery, and mRNA processing factors (for review see [12-14]). Several studies have established links between transcription elongation and mRNA export (for review see [15]). The TREX complex includes components involved in transcription elongation (the four subunits of the THO complex) and the essential mRNA export factors Sub2p and Yralp $[16,17]$. The interactions between Sub2p, Yra1p and Mex67p lead to a model of sequential binding where Sub2p is recruited by THO and brings Yralp that works as an adaptor of Mex67p interaction with the transcript [15,18]. Dbp5p also interacts physically with Yra1p in yeast cells and its ortholog, Aly/REF in mammalian cells [19]. Since Yra1p does not shuttle, it appears to be removed from the mRNP prior to its passage through the NPC [6]. We and others have demonstrated that loss-of-function mutations in some components of the basic transcription machinery are able to suppress the growth and mRNA export defects observed in $d b p 5$ or sub2 mutant strains $[11,20]$. One possible explanation for this is that a reduction in the transcription rate could enhance proper formation of mRNPs under condition when some of the proteins needed for mRNP biogenesis are compromised but still functioning $[11,21]$.

To further our understanding of the linkage between nuclear events of mRNA biogenesis and mRNA export, we investigated the effect of mutations affecting various components of the transcription initiation apparatus on the phenotypes of mRNA export mutant strains. Our results show that growth and mRNA export defects of $d b p 5$ and mex67 mutant strains can be suppressed by mutation of specific transcription initiation components, but suppression was not observed for mutants acting in the very first steps of the pre-initiation complex (PIC) formation. The data suggest that reducing the speed with which a transcription event converts from initiation and promoter clearance to elongation can have a positive effect on mRNP formation in dbp5 and mex67 mutant strains. We hypothesize that this occurs in mutant strains by permitting more effective recruitment to the nascent mRNP of key proteins that are either partiallyfunctional in mutant strains or of limiting abundance.

\section{Results}

The synthetic lethality of bur6 with dbp5 mutations can be suppressed by overexpression of a truncated allele of RNA pol II subunit RPB2

In previous work we identified a variety of genetic interactions between DBP5 and different factors involved in transcription initiation [11]. Mutations of $d b p 5$ were synthetically lethal with mutations in the gene encoding Bur6p [11]. Bur6p together with Ydr1p form the transcription repressor $\mathrm{NC2}$ [22]. In vitro, the $\mathrm{NC} 2$ complex interacts with TBP and blocks the association of TFIIA and TFIIB [22,23]. As can be observed in Figure $1 \mathrm{~A}$, bur6 mutations are also synthetically lethal with yra1-1 and sub2-85 but not with mex67-5 or mex67-6, although mex67 bur6 double mutants are impaired in growth and show a reduced viability (Figure 1A; results not shown).

We attempted to suppress the synthetic lethal phenotype of the double bur6- 1 rat $8-2 / d b p 5$ mutant by transforming the double mutant strain carrying the wild-type DBP5 gene on a URA3/CEN plasmid with a high-copy-number YEp13-based library. We selected transformants on SC-leucine plates and replica-plated these onto $5^{\prime}$-FOA containing plates. As expected, plasmids carrying the BUR6 wild-type gene were isolated $(\mathrm{n}=23)$, but no plasmids containing the DBP5 wild-type gene were recovered. Based on prior experiments using the same library (our unpublished results), we believe that the library lacks a plasmid containing DBP5. Besides plasmids containing BUR6, we isolated six independent clones carrying plasmids containing inserts of chromosome XV that included an N-terminal fragment of the $R P B 2$ gene, the YOR152c ORF and an N-terminal fragment of the PDR5 gene. By subcloning, we mapped the suppressor activity to the truncated $R P B 2$ gene. This allele encodes the first 379 amino acids (total length of 1,224 residues) and was named $r p b 2 t$.

The effect of rpb2t on the bur6-1 rat8-2 double mutant prompted us to determine whether expression of $r p b 2 t$ from a high copy plasmid would be able to suppress the growth defect shown by individual $d b p 5$ and bur6 mutants. The effect of Rpb2t on the growth defect caused by the depletion of Bur6p has been previously reported [24]. Figure 1B shows that Rpb2tp, but not wild-type Rpb2p, partially suppresses the growth defect of rat8-2 at $34^{\circ} \mathrm{C}$. However, when the YEp-rpb2t plasmid was introduced into a yeast strain where the DBP5 gene was expressed under the control of the doxycyclinerepressible tetO promoter, we did not observe any growth improvement in the presence of doxycycline (Figure 1B). Therefore, $r p b 2 t$ is not a bypass suppressor, since expression of Rpb2tp does not suppress the growth defect caused by depletion of Dbp5p. We also examined the effect of $r p b 2 t$ on other mutants affecting important mRNA export factors. Figure $1 \mathrm{~B}$ shows that $r p b 2 t$ improved the growth of the sub2-85 and yra1-1 mutants at a semi-restrictive temperature. However, it had no effect on the growth of the mex67-5 or mex67-6 strains (data not shown). Suppression by $r p b 2 t$ thus correlates with synthetic lethality with bur6-1 in that rat8-2, yra1-1 
A

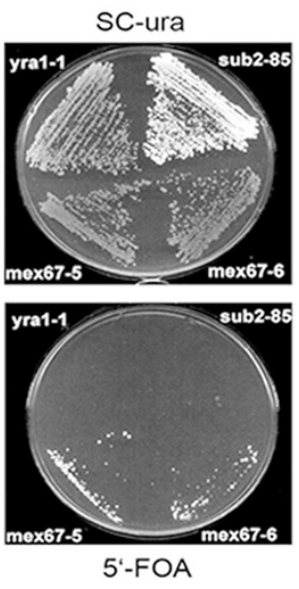

B

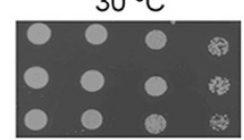

YPD

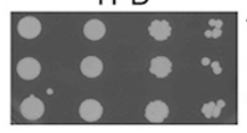

$30^{\circ} \mathrm{C}$

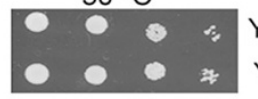

*

$30^{\circ} \mathrm{C}$

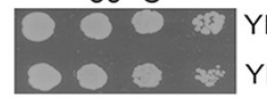

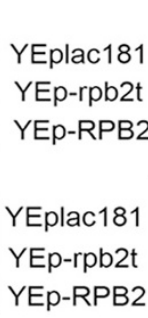

YEplac181

YEp-rpb2t

Eplac181 $34^{\circ} \mathrm{C}$

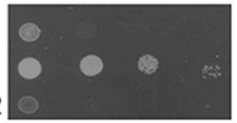

rat8-2

YPD + doxycycline

$P_{\text {teto-DBP5 }}$

$34^{\circ} \mathrm{C}$

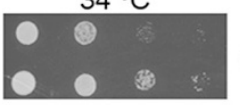

sub2-85

$34^{\circ} \mathrm{C}$

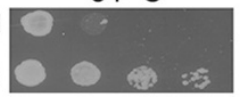

yra1-1

Figure 1 Genetic interactions among mutants affecting mRNA export factors Dbp5p/Rat8p, Mex67p, Sub2p and Yra1p, and the transcription factor Bur6p and truncated Rpb2tp. (A) Synthetic lethality between bur6-ts and yra 1-1 or sub2-85. Double mutants yra1-1 bur6-ts [pYRA1/URA3] (yra1-1 in the Figure), sub2-85 bur6-ts [pSUB2/URA3] (sub2-85 in the Figure), mex67-5 bur6-ts [pMEX67/URA3] (mex67-5 in the Figure) and mex67-6 bur6-ts [pMEX67/URA3] (mex67-6 in the Figure) were streaked on SC-ura (as control) or 5'-FOA (to analyze synthetic lethality) and incubated at $30^{\circ} \mathrm{C}$ for 4 days. (B) rat8-2 mutant was transformed with a C-terminal deletion of RPB2 carried on the multicopy plasmid YEplac181 (YEp-rpb2t), the wild type RPB2 gene cloned in YEplac181 (YEp-RPB2) or the empty vector (YEplac181). Serial dilutions (1:10) of transformed cells were spotted onto YPD plates and incubated for 3 days at different temperatures. A yeast strain carrying the DBP5 gene under the control of the regulatable promoter containing tetO (PtetO-DBP5) was transformed with the indicated plasmids. Transformed cells were spotted onto YPD or YPD containing $10 \mathrm{mg} / \mathrm{L}$ doxycycline and incubated for 3 days at $30{ }^{\circ} \mathrm{C}$. sub2-85 and yra1-1 mutants were transformed with the YEp-rpb2t plasmid or the empty vector YEplac181 and growth was analyzed by spotting serial dilutions of the transformants on YPD plates and incubation at different temperatures.

and sub2-85 all show synthetic lethality with bur6-1 but mex67-5 and mex67-6 do not.

\section{A reduction of the amount of RNA pol II does not suppress the growth defect of the rat8-2 mutant}

We considered the possibility that truncated Rpb2tp might cause a reduction in RNA pol II activity, as a result of the presence of non-functional RNA pol II holoenzymes (those containing the truncated Rpb2tp) competing with functional ones. To check if a reduction of the amount of different subunits of RNA pol II would be able to suppress the growth defect shown by the rat8-2 mutant at restrictive temperature, we tested strains in which the promoters of different RNA pol II subunits had been replaced by the regulatable tet $O$ promoter. Figure 2 shows that strains expressing the $R P B 1, R P B 2$ or $R P B 7$ genes under the control of the tet $O$ promoter were able to grow to different extents at low doxycycline concentration $(1 \mu \mathrm{g} / \mathrm{ml})$. However the reduction of RPB1, RPB2 and $R P B 7$ expression further impaired, rather than enhanced, the growth defect of the rat8-2 mutant at the semi-restrictive temperature. In addition, we observed that deletion of the non-essential $R P B 9$ gene also had a negative effect on the growth of rat8-2 at $34^{\circ} \mathrm{C}$ (data not shown). Therefore, these findings demonstrate that reducing the amount of RNA pol II is not sufficient to suppress the growth defect of rat8-2.
Genetic interactions of mRNA export factor mutants with components of RNA pol II general transcription factors (GTFs)

The suppression of dbp5/rat 8 mutations by mutations in SSL1, encoding a subunit of TFIIH [11] prompted us to analyze the genetic interactions of rat8-2 with other general transcription factors (GTFs). We replaced the promoter of the TFIIH-component TFB1 with the doxycycline-repressible promoter containing tetO. As shown in Figure 3A, the partial depletion of Tfb1p alleviated the growth defect of the rat $8-2$ mutant at $34^{\circ} \mathrm{C}$.

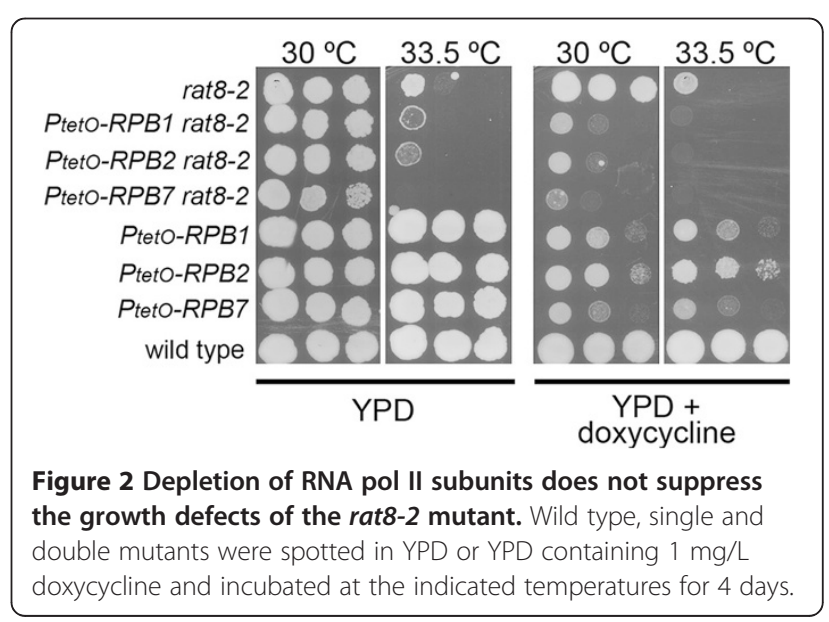



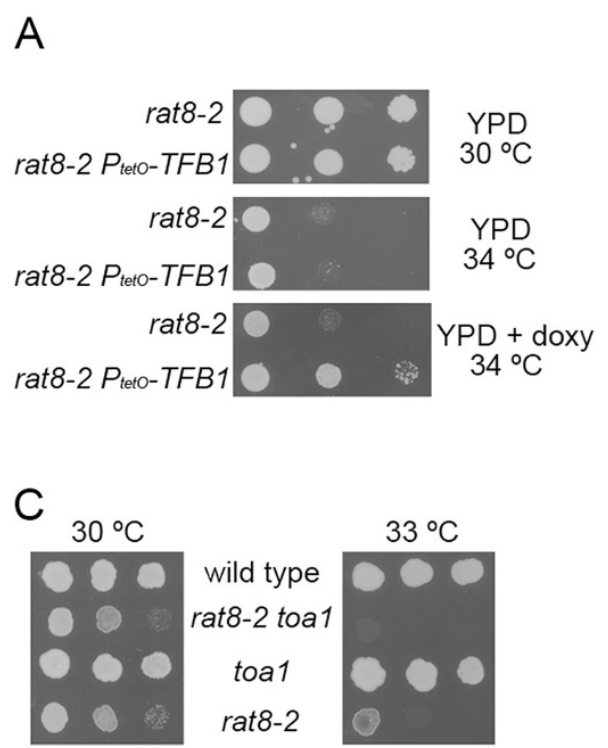

B

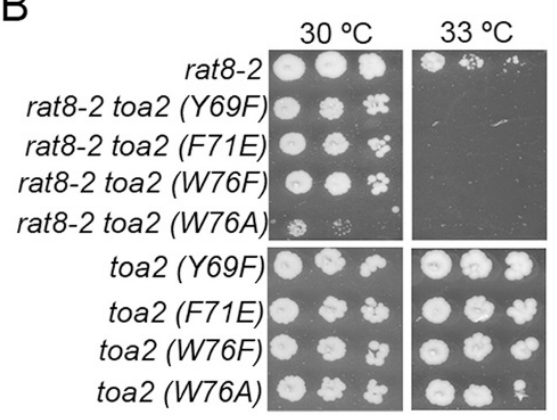

$\mathrm{D}$

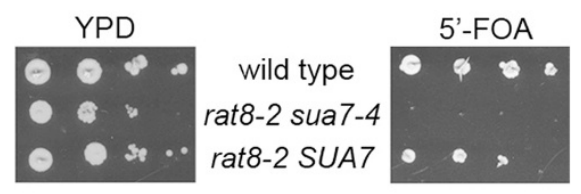

Figure 3 Genetic interactions between mRNA export factors and components of the transcription machinery. (A) Suppression of rat8-2 by depletion of the TFIIH component Tfb1 p. rat8-2 single mutant and rat8-2 $P_{\text {teto }}$-TFB1 double mutant were spotted onto YPD or YPD containing $10 \mathrm{mg} / \mathrm{L}$ of doxycycline plates and incubated at the indicated temperatures for 4 days. (B) Synthetic sickness of rat8-2 and toa2 mutations. Serial dilutions of single and double mutants strains were spotted onto YPD plates and incubated at different temperatures for 4 days. (C) Mutation in toa1 impairs growth of rat8-2 mutants. Strains analyzed were segregants from the cross CSY550 (rat8-2) x MY1870 (toa1-2). Cells were spotted onto a YPD plate and incubated for 3 days at the indicated temperatures. (D) Synthetic lethality between rat8-2 and sua7-4. Double mutants rat8-2 sua7:: LEU2 [pSUA7/URA3] carrying the wild type SUA7 gene or the mutant allele sua7-4 in a HIS3/CEN plasmid were spotted on YPD or 5/FOA plates and incubated at $30^{\circ} \mathrm{C}$ for 4 days.

Next, we analyzed the genetic interactions between rat8-2 and mutants affecting TFIIA and TFIIB. We tested the TFIIA components encoded by the TOA1 and $T O A 2$ genes. In the case of TOA1, we combined the rat8-2 mutation with toa1-2, an allele isolated as suppressor of a defect in NC2 function [25]. As it has been mentioned above, mutations of $d b p 5$ were found to be synthetically lethal with mutations in the gene encoding the NC2-component Bur6p. In the case of Toa2p, we used four different single point mutants of TOA2 [26]. Both rat8-2 toa2 (Figure 3B) and rat8-2 toa1 (Figure 3C) double mutants grew notably more slowly at semirestrictive temperature than the single mutants. Similarly, we observed that rat8-2 was synthetically lethal at $30^{\circ} \mathrm{C}$ with a temperature sensitive allele of SUA7 (sua7-4), encoding TFIIB [27], although both single mutants grew well at $30^{\circ}$ (Figure 3D and data not shown).

Taken together, these genetic analyses indicate that the pattern of synthetic lethality and suppression cannot be explained by the simple model related to increasing or decreasing the overall amount of transcription.

\section{Genetic interaction between ssl1 and mex67 mutants}

Mutations in SSL1 partially suppress the growth defect of rat8-2 at semi-permissive temperatures but not at the restrictive temperature of $37^{\circ} \mathrm{C}$ (Figure 4A; [11]). We combined the thermosensitive ssl1-1 allele with yra1-1, sub2-85 and mex67-5 and analyzed the growth of the double mutants at the restrictive temperature of $37^{\circ} \mathrm{C}$. As can be observed in Figure 4A, the combination of the wild type SSL1 and the ssl1-1 mutant allele partially suppressed the growth defect of the mex67-5 mutant at $37^{\circ}$ $\mathrm{C}$ although suppression was not observed if only ssl1-1 was present, presumably because ssl1-1 cells cannot grow at $37^{\circ} \mathrm{C}$ [28] and mex67-5 cannot suppress its growth defect. A similar result was found for mex67-6, which has a stronger growth defect than mex67-5 (Figure 4B). In this case, the double mutant mex67-6 ssl1-1 grew slightly better than the single mex67-6 mutant at $34^{\circ} \mathrm{C}$. The presence of wild type SSL1 in addition to ssl1-1 improved growth of mex67-6 at $34^{\circ} \mathrm{C}$, but cells were still unable to grow at $37^{\circ} \mathrm{C}$ (Figure 4B).

The unexpected effect of having the simultaneous presence of both wild-type and mutant Ssl1p prompted us to examine how suppression was affected by altering the relative copy numbers of wild type and mutant alleles of SSL1. We transformed the mex67-5 ssl1-1 double mutant strain with a CEN (pSSL1) or $2 \mu \mathrm{m}$ plasmid (pPD5) carrying SSL1. Growth of these transformants at different temperatures is shown in Figure 4B. As can be observed, the wild-type SSL1 gene was required for suppression and suppression was stronger when cells contained fewer copies of the wild type SSL1 gene (compare growth with pSSL1 and pPD5). 


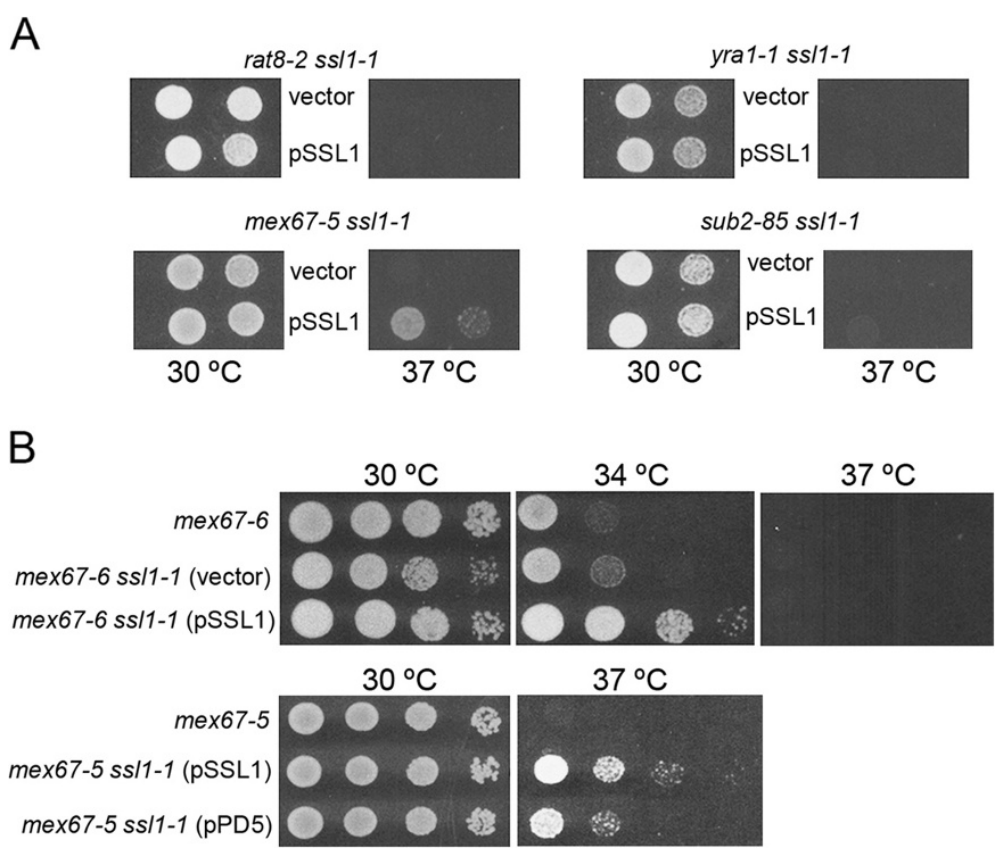

Figure 4 Suppression of mex67 ts growth defect by ss/1-1. (A) rat8-2, yra1-1, mex67-5 and sub2-85 mutations were combined with ss/1-1. Double mutant strains were transformed with a CEN plasmid containing the SSL1 wild type gene (pSSL1), or the empty vector pRS316 (vector). Serial dilutions (1:10) were spotted onto SC-ura plates and incubated for 3 days at different temperatures. (B) Suppression of mex67-6 ts growth defect by ss/1-1. Serial dilutions (1:10) of mex67-6 single mutant and mex67-6 ss/7-1 double mutant (either transformed with pSSL1 or the empty vector pRS316) were spotted onto YPD plates and incubated for 3 days at different temperatures. Effect of the copy number of the SSL1 wild type gene on growth of the mex67-5 ss/1-1 double mutant strain. Serial dilutions (1:10) of mex67-5 single mutant and mex67-5 ss/1-1 double mutant transformed with SSL1 in a CEN/URA3 plasmid (pSSL1) or in a multicopy vector (PPD5) were spotted onto YPD plates and incubated for 3 days at different temperatures.

\section{Mutation of SSL1 suppresses other phenotypes of the mex67-5 mutant}

We previously observed that, at semi-permissive temperature of $34^{\circ} \mathrm{C}$, rat8-2 ssl1-1 double mutant cells accumulated significantly less poly $(\mathrm{A})^{+} \mathrm{mRNA}$ in nuclei than did the single rat8-2 mutant [11]. Since mex67 mutants have a strong defect in export of poly $(\mathrm{A})^{+}$RNA at elevated temperatures [29] we examined how the ssl1-1 mutation would affect the mRNA export defect of the mex67 mutants. Figure 5 shows that ssl1-1 altered the distribution of poly $(\mathrm{A})^{+}$mRNA in mex67-5 cells. More poly(A) ${ }^{+}$RNA was cytoplasmic in the ssl1-1 mex67-5 double mutant than in mex67-5 cells. In addition, although mRNA still accumulated in the nucleus, it was mainly distributed around the nuclear periphery in double-mutant cells while poly(A) ${ }^{+}$RNA filled the nucleus in mex67-5 cells. When the ssl1-1 mex67-5 double mutant was transformed with the wild type SSL1 gene on a centromeric plasmid, there was less suppression of the mex67-5 mRNA export defect, although these cells also showed a stronger cytoplasmic signal for poly(A) ${ }^{+}$RNA than did mex67-5 cells. In addition, introduction of SSL1 eliminated the perinuclear distribution and poly $(\mathrm{A})^{+}$mRNA could be seen throughout the nucleus (Figure 5). The fact that $\mathrm{SSL}^{+}$is required for growth of mex67-5 ssl1-1 but reduces suppression of the mex67-5 mRNA export defect by ssl1-1 suggests that mutant Ssl1-1p protein is responsible for reducing the export defect of mex67-5 but wild type Ssl1p is required for provision of some Ssl1p function(s) compromised by the ssl1-1 mutation (see discussion below).

Previous work has also shown that Dbp5p accumulates in nuclei in several strains with defects in nuclear transport, including mex67-5 [3]. We found that the nuclear accumulation of Dbp5-GFP observed in the mex67-5 mutant at $37^{\circ} \mathrm{C}$ was prevented completely by the ssll-1 mutation (Figure 6). However, when the double-mutant mex67-5 ssl1-1 strain was transformed with wild-type SSL1 on a centromeric plasmid, we observed an intermediate effect: there was less of an increase in cytoplasmic Dbp5-GFP (although cytoplasmic staining can also be observed at $23^{\circ} \mathrm{C}$ ) and more nuclear Dbp5-GFP than when wild-type $S S L 1$ was not present (Figure 6).

It has been reported that mutations in MEX67 and other genes encoding proteins involved in mRNA export, including NUP159, GLE1 and DBP5, produce nascent transcripts carrying poly $(\mathrm{A})^{+}$tails roughly 30 residues longer than those observed in wild type cells [30]. We analyzed the effect of the ssl1-1 mutation on the length of poly $(\mathrm{A})^{+}$tails produced in mex67-5 cells 


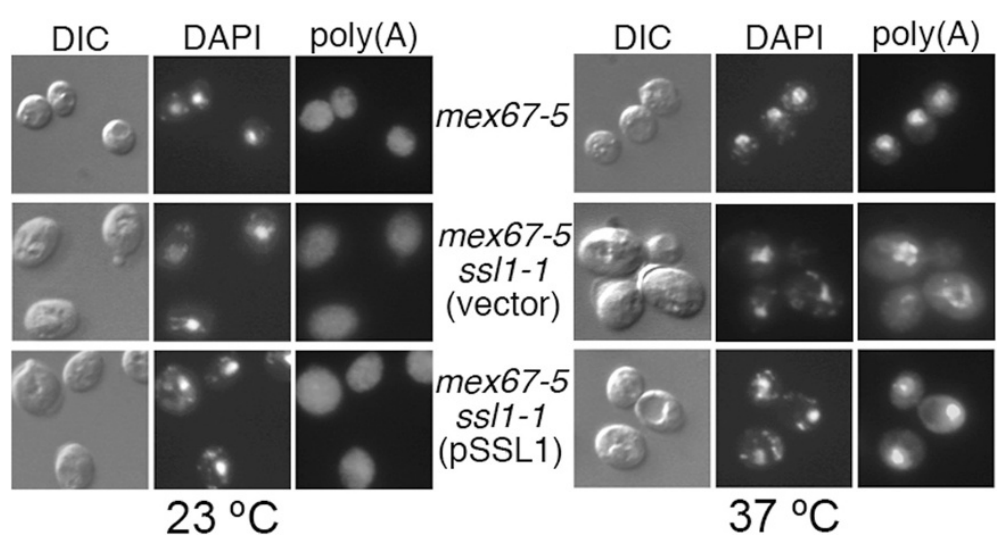

Figure 5 ss/1-1 mutation affects nuclear accumulation of poly(A) ${ }^{+}$RNA in mex67-5 mutant cells. Single mex67-5 and double mex67-5 ss/7-1 [either transformed with the empty vector (vector) or SSL1 in a CEN plasmid (pSSL1)] mutant cells were grown to mid-log phase at $23^{\circ} \mathrm{C}$ and incubated for 1 hour at $37^{\circ} \mathrm{C}$. In all the cases, cells were fixed and in situ hybridization was performed using a digoxigenin-conjugated oligo(dT) probe, followed by incubation with a FITC-conjugated anti-digoxigenin antibody [poly(A)]. DNA was visualized by DAPI staining.

(Figure 7A). At restrictive temperature, the amount of poly $(\mathrm{A})^{+}$RNA produced was reduced in the mex67-5 ssl1-1 mutant strain (Figure 7A, lane 6) compared to mex67-5 (lane 4) or wild-type cells (lane 2), consistent with the importance of Ssl1p and TFIIH for transcription. However, although some mRNAs with extended poly $(\mathrm{A})^{+}$tails were still produced in the mex67-5 ssl1-1 double mutant, the distribution of poly $(\mathrm{A})^{+}$tail lengths was shifted towards a wild-type distribution (Figure 7B). When the mex67-5 ssl1-1 double mutant strain was transformed with the wild type SSL1 gene, a wild-type level of transcripts was recovered at restrictive temperature (Figure 7A, lane 8), but there was considerably less of a shift towards poly $(\mathrm{A})^{+}$tails of normal length than when wild-type SSL1 was not present (Figure 7B).

\section{Discussion}

Genetic interactions among transcription and export factors suggest complex relationships between transcription and export activities

Previous studies showed that mutations in SSL1 and RAD3, encoding TFIIH components, and CEG1, encoding one of the enzymes of the capping apparatus, result in suppression of the growth and mRNA export defects observed in some mRNA export factor mutant strains $([11,20,31]$, see above). In this report we describe

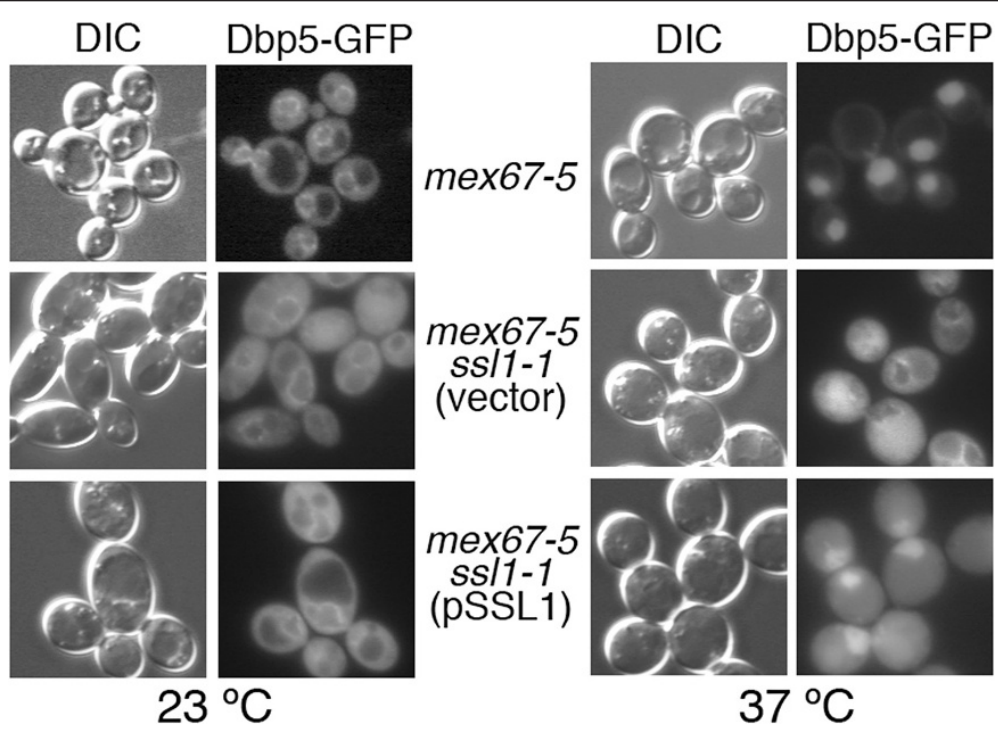

Figure 6 ss/1-1 mutation affects cellular distribution of Dbp5-GFP in mex67-5 mutant cells. Single mex67-5 and double mex67-5 ss/1-1 [either transformed with the empty vector (vector) or SSL1 in a CEN plasmid (pSSL1)] mutant cells were transformed with plasmid pCS835 (expressing a Dbp5-GFP fusion). Cells were grown to mid-log phase at $23^{\circ} \mathrm{C}$ and incubated for 10 minutes at at $37^{\circ} \mathrm{C}$. 
A

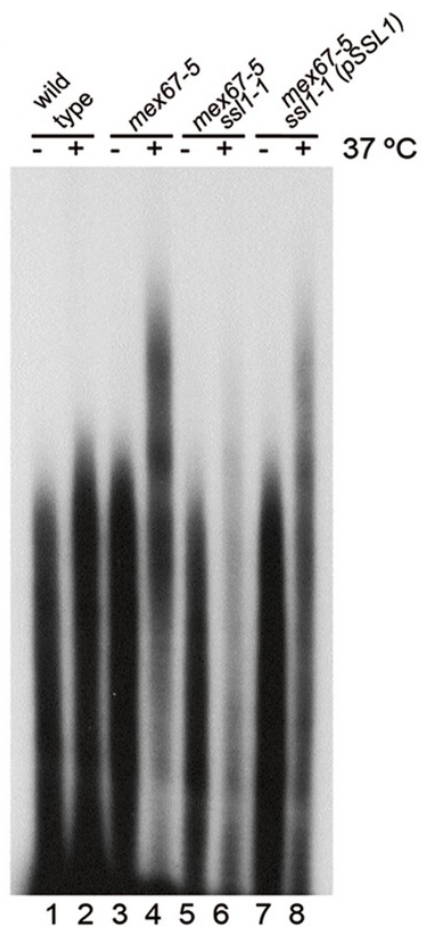

B

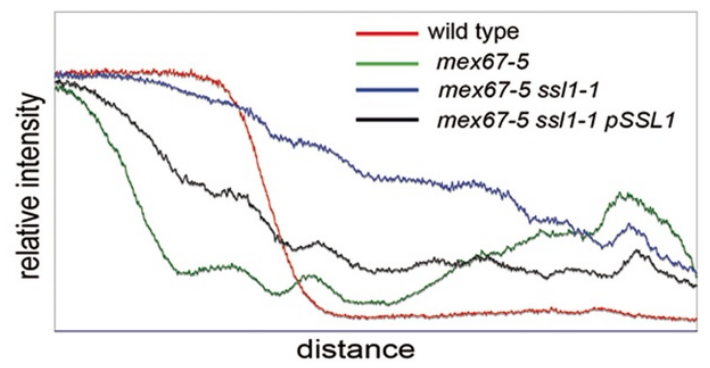

Figure 7 ss/1-1 mutation affects hyperpolyadenylation in mex67-5 mutant cells. (A) Poly(A) tail length was determined by isolating total RNA from each strain that had been grown continuously at $23^{\circ} \mathrm{C}(-)$ or shifted to $37^{\circ} \mathrm{C}$ for $30 \mathrm{~min}(+)$. The resulting RNAs were digested to completion with RNases $T_{1}$ and $A$. The remaining oligo(A) and poly $(A)$ fragments were then end labeled with $\left[{ }^{32} \mathrm{P}\right] p C p$ and RNA ligase and resolved on 9\% polyacrylamide-7 M urea-TBE gels Gels were dried and exposed to X-Omat Blue film. (B) Densitometric analysis of the poly(A) tail length. Lanes corresponding to the samples obtained at restrictive temperature $\left(37^{\circ} \mathrm{C}\right)$ were densitometered using the Quantity One program (Bio-Rad).

additional genetic interactions between mutations affecting components of the basal transcription machinery and rat8-2, sub2-85, yra1-1, mex67-5 and mex67-6. In addition, mutations in DBP5, YRA1 and SUB2, and $M E X 67$, are synthetically lethal (or synthetically sick) with mutations in the gene encoding the TBP-binding protein Bur6p (Figure 1A). We also report the suppression of rat8-2 mutation by partial depletion of Tfb1p (a TFIIH component) and by expression of a truncated form of Rpb2p (referred as Rpb2tp), which is also able to suppress the growth defects of the yra1-1 and sub2-85 mutants (Figure 1B).

We and others have suggested that mutations affecting components of the basal transcription machinery suppress defects in mRNA export factors by reducing the rate of formation of mRNP complexes [11,20]. A key question is whether simply reducing the overall rate of mRNP production is sufficient to suppress these defects. The finding that loss-of-function mutations affecting some components of the basic transcription apparatus enhance the growth defect of mRNA export mutants (Figure 3) suggests that mere reduction in the production of mRNP is not sufficient for suppression. The list of mutations without suppressive effect on rat8-2 includes a mutant allele of SPT6 (encoding a nucleosome remodeling protein that functions in transcription elongation), a disruption of ELP3 (encoding a subunit of the elongator complex) or HPR1 (encoding a subunit of THO/TREX) [11] and alleles affecting the TFIIA (Toalp and Toa2p) and TFIIB (Sua7p) components. Elp3p, Spt6p, and Hpr1p have been reported to be involved in transcriptional elongation [32-34]. TFIIA and TFIIB are general transcription factors recruited early during preinitiation complex formation. On the other hand, suppression of rat8-2 has been observed with mutations in two different subunits of the TFIIH complex (Ssl1p and Tfblp), the capping enzyme Ceg1p and by expression of the truncated Rpb2tp. TFIIH plays a critical role in the formation of the open complex during transcription initiation and in promoter escape, and it has been proposed that the capping enzyme executes a checkpoint during early transcription that assesses whether capping of the nascent transcript has occurred within a specific time window, and if so, allowing elongation to proceed [35-37]. Thus, the pattern of genetic interactions (both suppressive and not suppressive) we have described suggests that suppression occurs with mutants affecting events after formation of the preinitiation complex but prior to the onset of extensive 
transcription elongation. These results may indicate that only a limited time window exists for co-transcriptional loading of mRNA export factors onto the nascent transcript. By this scenario, truncated Rpb2tp might act by sequestering some component(s) of the transcription apparatus, resulting in reduced efficiency of some early step of transcription, thereby permitting the partiallydefective mutant mRNA export factor to perform its function sufficiently well to support growth.

\section{Different phenotypes of the mex67 mutant strain can be suppressed by the combination of wild type and mutant SSL1 alleles}

In a previous report we showed that some defects of the rat8-2 mutant could be suppressed by mutations in general transcription factors such as the TFIIH component SSL1 [11]. Similarly, Jensen et al. [38] found that mutations in $R A D 3$, also encoding a TFIIH component, suppressed both growth and mRNA export defects of the sub2-201 mutant strain. In the studies reported here, we analyzed the effect of the ssl1-1 mutation on different export factor mutant strains. Our results show that, as occurs with DBP5, ssl1-1 partially suppresses the growth defect of the mex67-6 mutant at the semi-restrictive temperature of $34^{\circ} \mathrm{C}$ (Figure $4 \mathrm{~B}$ ). The mex67-5 mutant strain is able to grow at $34^{\circ} \mathrm{C}$ but not at $37^{\circ} \mathrm{C}$. However, this restrictive temperature could not be used to check the suppression of mex67-5 by ssl1-1, since the ssl1-1 mutation prevents growth at $37^{\circ} \mathrm{C}$ [28]. Unexpectedly, we observed partial suppression of the mex67-5 growth defect at $37^{\circ} \mathrm{C}$ when we simultaneously introduced the recessive ssl1-1 mutant allele and the wild-type SSL1 gene on a centromeric plasmid (Figure 4B). The suppression caused by the ssl1-1/SSL1 combination was also observed for the mex67-6 mutant strain, and is sensitive to the ratio of wild type and mutant Ssl1p (Figure 4B).

As occurs with rat8-2 mutants [11], the ssl1-1 mutation alters the distribution of poly $(\mathrm{A})^{+}$mRNA in mex675 cells (Figure 5). Interestingly, suppression of the mRNA export and hyper-polyadenylation defects of mex67-5 by ssl1-1 was reduced if wild-type SSL1 was also present. Our data suggest that wild-type Ssl1p is needed because the Ssl1-1p protein cannot perform all of Ssl1p's functions at $37^{\circ} \mathrm{C}$, although the suppression of mex67-5 mutant phenotypes by Ssl1-1p indicates that it is performing some functions. The finding that the presence of wild type Ssl1p reduces the degree of suppression of the mRNA export and hyper-polyadenylation defects of mex67-5 by Ssl1-1p suggests that there may be important difference in mRNPs produced through the action of TFIIH that contains mutant as opposed to wild-type Ssl1p. Perhaps key events related to recruitment of important export factors occur during the period that Ssl1p/TFIIH functions and only those mediated by TFIIH containing mutant Ssl1-1p occur in a manner that permits a later effective recruitment of Mex67-5p. We propose that suppression of the hyperpolyadenylation defect by Ssl1-1p reflects the fact that those mRNPs produced through the action of Ssl1-1pcontaining TFIIH undergo more accurate $3^{\prime}$ processing than those produced in mex67-5 cells (where wild-type Ssl1p is present), thereby reducing hyper-polyadenylation and increasing mRNA export. However, mRNA still accumulates in nuclei in mex67-5/ssl1-1 cells (although accumulation is lower than in mex67-5 cells). Furthermore, in the presence of ssl1-1 but not SSL1, the distribution of the poly $(\mathrm{A})^{+}$signal inside the nucleus differs from that observed in the mex67-5 single mutant, with $\operatorname{poly}(\mathrm{A})^{+}$mRNA accumulating primarily at the nuclear periphery in mex67-5/ssl1-1 cells (Figure 5). The accumulation of poly $(\mathrm{A})^{+}$RNA in nuclear foci observed in the mex67-5 mutant is thought to reflect retention of mRNA near sites of transcription, whereas the accumulation at the nuclear periphery observed in the mex67-5 ssl1-1 strain suggests that the ssl1-1 mutation permitted release of poly $(\mathrm{A})^{+}$mRNP from intranuclear sites, possibly reflecting an improvement in the quality of the mRNP produced. The fact that a partial mRNA export block remains in mex67-5/ssl1-1 cells could reflect the fact that the presence of ssl1-1 does not return the accuracy of mRNP production to wild-type levels and that some mRNPs are not in an exportable configuration. Alternatively, it may be that the Mex67-5p protein is less functional than wild type Mex67p for functions that occur during translocation through the NPC. Since mex67-5/ssl1-1 cells grow, there must be an increase in mRNA export. This is more easily explained by improved quality of the mRNP due to Ssl1-1p than to Ssl1-1p acting to enhance Mex67-5p functionality during NPC translocation, since Ssl1p is not part of the exported mRNA and is not expected to have any direct affect on the actual mRNA export event. It is interesting that a modest increase in the amount of cytoplasmic poly $(\mathrm{A})^{+}$ RNA allows mex67-5/ssl1-1 cells to grow whereas mex67-5 cells do not. At present, it is not technically possible to determine if there are important qualitative changes in the organization or composition of mRNPs when the ssl1-1 allele is present. In a large number of experiments over many years and in different mutants we have observed that cellular growth does not require levels of cytoplasmic poly(A) ${ }^{+}$RNA equal to or even near what is observed in wild-type cells. Often cells with substantial nuclear accumulation of poly $(\mathrm{A})^{+}$RNA and a modest cytoplasmic signal are able to grow, albeit at a reduced rate in many cases. This is clearly the case with the nup $159 \Delta \mathrm{N}$ mutant which grows at a moderate rate at $25^{\circ} \mathrm{C}$ despite strong accumulation of poly $(\mathrm{A})^{+}$RNA in nuclei [3]. 
Nuclear accumulation of Dbp5-GFP also occurs in mex67-5 cells [3]. This accumulation is not observed in the double mex67-5 ssl1-1 mutant, whereas in the mex67-5 ssl1-1/SSL1 strain, there is a strong nuclear signal, although accompanied by an increased cytoplasmic staining, relative to that seen in mex67-5 cells (Figure 6). Although the causes for the nuclear accumulation of Dbp5p in mex67 remain to be clarified, our results suggest that this phenotype could be related to mRNP retention at transcription site foci due to the mex67-5 mutation. These mRNPs would contain Dbp5-GFP resulting in accumulation of Dbp5-GFP in nuclei. The nuclear accumulation of Dbp5p could also contribute to the hyper-polyadenylation defect since this may lead to reduced levels of Dbp5p associated with NPC filaments, where it functions to mediate remodeling of the mRNP complex. In this situation, removal of shuttling mRNA binding proteins by Dbp5p might be less efficient and the mRNPs produced might have a defective composition due to sub-optimal levels of shuttling mRNAbinding proteins that become part of mRNPs in the nucleus. Additional work will be required to elucidate how Dbp5p participates in the assembly of an export competent mRNP and what distinguishes export-competent mRNPs from those that accumulate in nuclei.

\section{Conclusions}

Our previous results and those reported here suggest roles for Dbp5p both during transcription and at the NPC during mRNP translocation. The finding that mutations in components of TFIIA and TFIIB or a reduction in the amount of RNA pol II exacerbate the growth defect of $d b p 5 /$ rat8-2 at semi-restrictive temperature indicates that mere reduction in the amount of mRNP produced is not sufficient to suppress the defects caused by a defective Dbp5p. The data suggest that suppression occurs only with mutants affecting events within a narrow window of the mRNP biogenesis process. We propose that reducing the speed with which transcription converts from initiation and promoter clearance to elongation may have a positive effect on mRNP formation in $d b p 5$ mutant strains by permitting more effective recruitment of $\mathrm{mRNP}$ proteins to the nascent mRNP.

\section{Methods}

\section{Yeast strains and genetic methods}

Yeast strains used in this study are listed in Table 1. Strains were grown using standard methods. For growth assays, yeast cells were diluted to the same $\mathrm{OD}_{600}$, and serial dilutions (1:10) were spotted onto YPD or selective plates and incubated at various temperatures. $5^{\prime}$ fluoroorotic acid ( $5^{\prime}$-FOA) was added to synthetic complete media at $1 \mathrm{~g} / \mathrm{L}$. Doxycycline was added to YPD plates at 1 or $10 \mathrm{mg} / \mathrm{L}$.

\section{High copy suppression screen}

Multicopy suppressors of the synthetic lethality between bur6-1 and rat8-2 mutant alleles were obtained by transforming the bur6-1 rat8-2 double mutant strain carrying the wild type DBP5 gene in a URA3/CEN plasmid (pCS831) with a yeast genomic library cloned in the YEp13 vector (LEU2/2 $\mu \mathrm{m})$. Transformants $(40,000)$ were grown on selective medium at $30^{\circ} \mathrm{C}$ for 3 days and then replica plated onto $5^{\prime}$-FOA-containing plates and incubated two additional days at $30^{\circ} \mathrm{C}$. Plasmids able to suppress the synthetic lethality were isolated and used to re-transform the bur6-1 rat8-2 double mutant. For the positive clones, insert ends were determined by DNA sequencing, revealing that six of them were identical. In these plasmids, the suppressor activity was mapped to a $X b a I$ fragment (one of the XbaI sites in the YEp13 vector and the second in the genomic insert) that included sequences corresponding to the promoter and $5^{\prime}$-terminal part of the RPB2 gene. The $\mathrm{Xbal}$ fragment was subcloned into the YEplac181 yielding the YEp-rpb2t plasmid.

\section{Plasmids}

The plasmid overexpressing wild type RPB2 gene has been described previously [24]. Plasmid pPD5 (containing the SSL1 gene in a multicopy vector) has been described previously [11]. To clone the SSL1 gene in a centromeric plasmid, an EagI-BamHI fragment from pPD5 was inserted between the Eagl and BamHI sites of the pRS314 or pRS316 vectors [41]. Plasmid pCS835 expressing a Dbp5-GFP fusion has been described [3].

\section{In situ hybridization and microscopy}

To localize poly $(\mathrm{A})^{+}$RNA within cells, we used in situ hybridization with an oligo $(\mathrm{dT})_{50}$ probe coupled to digoxigenin, which was performed as described previously [42]. Fluorescence images were examined and photographed using an Axiophot microscope (Zeiss) and a CCD camera.

\section{poly $(A)$ tail length measurements}

poly $(\mathrm{A})^{+}$tail length measurements was performed as described by [43]. Briefly, $10 \mu \mathrm{g}$ of total RNA was digested with $2 \mu \mathrm{g}$ of bovine pancreatic RNase per $\mathrm{ml}$ and 1,000 $\mathrm{U}$ of RNase T1 per $\mathrm{ml}$ to produce a RNA population consisting only of poly(A) tails. Each RNA sample was then end labeled with $100 \mu \mathrm{Ci}$ of $\left[5^{\prime}-{ }^{32} \mathrm{P}\right]$ cytidine $3^{\prime}, 5^{\prime}$ bis(phosphate) (NEN Life Science Products, Boston, Mass.) in a solution containing $50 \mathrm{mM}$ Tris$\mathrm{HCl}$ (pH 7.9), $15 \mathrm{mM} \mathrm{MgCl}_{2}, 3.3 \mathrm{mM}$ dithiothreitol (DTT), 2\% (vol/vol) dimethyl sulfoxide, $10 \mathrm{mg}$ of bovine serum albumin per $\mathrm{ml}, 25 \mu \mathrm{M}$ ATP, and $10 \mathrm{U}$ of 
Table 1 Yeast strains used in this study

\begin{tabular}{|c|c|c|}
\hline$\underline{\underline{\text { Strain }}}$ & Genotype & Source \\
\hline FY23 & MATa leu2 $\Delta 1$ ura3-52 trp $1 \Delta 63$ & [39] \\
\hline FY86 & MATa leu2 $\Delta 1$ ura3-52 his $3 \Delta 200$ & [39] \\
\hline CSY550 & MATa leu2 $\Delta 1$ ura3-52 trp $1 \Delta 63$ rat8-2 & [8] \\
\hline PDY4 & MATa ura3-52 his3 $\Delta 200$ ss/1-1 rat8-2 & [11] \\
\hline CSY564 & MATa leu2A1 ura3-52 his3 $\Delta 200$ rat8-2 [pRAT8.31] & [11] \\
\hline GY215 & 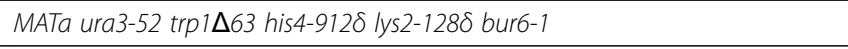 & G. Prelich \\
\hline PDY6 & MATa leu2A1 ura3-52 lys2-1288 rat8-2 bur6-1 [pRAT8.31] & [11] \\
\hline CSY560 & MATa leu2 $\Delta 1$ ura3-52 trp $1 \Delta 63$ rat8-2 [pRAT8.31] & [11] \\
\hline CS41-4.3 & MAT? ura3-52 leu2-3 his3-11 trp1-1 ade2-1 bur6:: HIS3 [pbur6-ts/CEN/LEU2] & D. Reinberg \\
\hline YMH2O2 & MAT? ura3-52 leu2-3 his3-11 trp1-1 ade2-1 bur6:: HIS3 [pBUR6/CEN/URA3] & D. Reinberg \\
\hline mex67-5 & MATa ade2 leu2 ura3 his3 trp1 mex67::HIS3 [pmex67-5/TRP1/CEN] & [29] \\
\hline yra1-1 & MATa ade2 leu2 ura3 his3 trp1 yra1::HIS3 [pyra1-1/TRP1/CEN] & [18] \\
\hline sub2-85 & MATa leu2 ura3 his3 trp1 sub2::kanMX4 [psub2-85/TRP1/CEN] & [40] \\
\hline yra1-1 bur6-ts & $\begin{array}{l}\text { MAT? ade2-1 trp1 ura3 his3 leu2 bur6:: HIS3 yra1::HIS3 [pbur6-ts/CEN/LEU2] } \\
\text { [pyra1-1/TRP1/CEN] [pYRA1/URA3/CEN] }\end{array}$ & This study \\
\hline sub2-85 bur6-ts & $\begin{array}{l}\text { MAT? trp1 ura3 his3 leu2 bur6::HIS3 sub2::kanMX4 [pbur6-ts/CEN/LEU2] } \\
\text { [psub2-85/TRP1/CEN] [pSUB2/URA3/CEN] }\end{array}$ & This study \\
\hline mex67-5 bur6-ts & $\begin{array}{l}\text { MAT? ade2-1 trp1 ura3 his3 leu2 bur6::HIS3 mex67::HIS3 [pbur6-ts/CEN/LEU2] } \\
\text { [pmex67-5/TRP1/CEN] [pMEX67 URA3/CEN] }\end{array}$ & This study \\
\hline mex67-6 bur6-ts & $\begin{array}{l}\text { MAT? ade2-1 trp1 ura3 his3 leu2 bur6::HIS3 mex67::HIS3 [pbur6-ts/CEN/LEU2] } \\
\text { [pmex67-6/TRP1/CEN] [pMEX67 URA3/CEN] }\end{array}$ & This study \\
\hline $\operatorname{mex} 67-5$ ss/1-1 & MAT? ade2 trp1? leu2 ura3 ss/1-1 mex67::HIS3 [pmex67-5/TRP1/CEN] & This study \\
\hline mex67-6 ss/1-1 & MAT? ade2 trp1? leu2 ura3 ss/1-1 mex67::HIS3 [pmex67-6/TRP1/CEN] & This study \\
\hline sub2-85 ss/1-1 & MAT? his3 ura3 sub2::kanMX4 [psub2-85/TRP1/CEN] & This study \\
\hline TOA1 rat8-2 & MAT? leu2 his3 ura3 rat8-2 & This study \\
\hline toa1-2 rat8-2 & MAT?leu2 trp1 rat8-2 toa1-2 & This study \\
\hline toa1-2 DBP5 & MAT? leu2 toal-2 & This study \\
\hline TOA1 DBP5 & MAT? leu2 his3 & This study \\
\hline MY1870 & MATa trp1 ura3-52 leu2::PET56 gal2 gcn4D toa1-2 & [25] \\
\hline toa2 (Y68F) & MATa toa2::HIS3 ade1 ura3-52 leu2A1 [ptoa2(Y68F)/LEU2/CEN] & [26] \\
\hline toa2 (F71E) & MATa toa2::HIS3 adel ura3-52 leu2A1 [ptoa2(F71E)/LEU2/CEN] & [26] \\
\hline toa2 (W76F) & MATa toa2::HIS3 ade1 ura3-52 leu2A1 [ptoa2(W76F)/LEU2/CEN] & [26] \\
\hline toa2 (W76A) & MATa toa2::HIS3 ade1 ura3-52 leu2 $\Delta 1$ [ptoa2(W76A)/LEU2/CEN] & [26] \\
\hline rat8-2 toa2 (Y68F) & 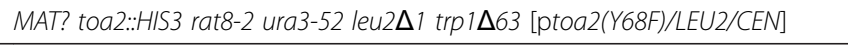 & This study \\
\hline rat8-2 toa2 (F71E) & 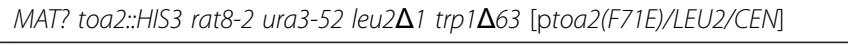 & This study \\
\hline rat8-2 toa2 (W76F) & MAT? toa2::HIS3 rat8-2 ura3-52 leu2 $\Delta 1$ trp1 $\Delta 63$ [ptoa2(W76F)/LEU2/CEN] & This study \\
\hline rat8-2 toa2 (W76A) & 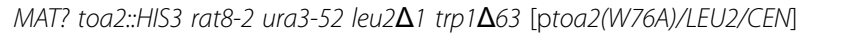 & This study \\
\hline
\end{tabular}

T4 RNA ligase for $21 \mathrm{~h}$ at $4^{\circ} \mathrm{C}$. RNA samples were then extracted with phenol and precipitated with ethanol. Pellets were then resuspended in a solution containing 96\% formamide, $0.1 \%$ bromophenol blue, and $0.1 \%$ xylene cyanol, denatured by boiling, and resolved on a $10 \%$ polyacrylamide-1 M urea-TBE (Tris-borate-EDTA) gel. Gels were dried and exposed to X-Omat Blue film (Kodak, Rochester, N.Y.).

\section{Abbreviations}

NPC: Nuclear pore complex; CTD: Carboxi-terminal domain; PIC: Pre-initiation complex; mRNP: Messenger ribonucleoprotein particle.

\section{Competing interests}

The authors declare that they have no competing interests.

\section{Authors' contributions}

FE carried out most of the experiments reported in the manuscript, participated in the design of the experiments, and wrote the paper. $\mathrm{CH}, \mathrm{NG}$ $\mathrm{N}, \mathrm{LP}-\mathrm{C}$ and $\mathrm{CVH}$ carried out some experiments. CNC conceived the study and wrote the paper. All authors read and approved the final manuscript.

\section{Acknowledgments}

We thank M. Collart, K. Weis, T. Donahue, A. Aguilera, G. Prelich, R. Young, D. Reinberg for providing plasmids and yeast strains, S. Brettschneider for technical assistance, and Jack Scarcelli for discussions and critical reading of the manuscript. This work was supported by grants from the Spanish 
Ministry of Education and Science (BFU2008-04082-C02-01/BMC and BFU2011-23501/BMC) and from Generalitat Valenciana (ACOMP2011/233) to F.E and a grant (GM33998) from the National Institute of General Medical Sciences of the N.I.H to C.N.C.

\section{Author details}

${ }^{1}$ Departamento de Bioquímica y Biología Molecular, Universitat de Valencia, Burjassot, Spain. ${ }^{2}$ Departments of Biochemistry and Genetics, Geisel School of Medicine at Dartmouth, Hanover, New Hampshire, NH, USA.

Received: 29 May 2012 Accepted: 31 August 2012

Published: 10 September 2012

\section{References}

1. Wente SR, Rout MP: The nuclear pore complex and nuclear transport. Cold Spring Harb Perspect Biol 2010, 2(10):a000562.

2. Hetzer MW, Wente SR: Border control at the nucleus: biogenesis and organization of the nuclear membrane and pore complexes. Dev Cell 2009, 17(5):606-616.

3. Hodge CA, Colot HV, Stafford P, Cole CN: Rat8p/Dbp5p is a shuttling transport factor that interacts with Rat7p/Nup159p and Gle1p and suppresses the mRNA export defect of xpo1-1 cells. EMBO J 1999, 18(20):5778-5788.

4. Hodge CA, Tran EJ, Noble KN, Alcazar-Roman AR, Ben-Yishay R, Scarcelli J J Folkmann AW, Shav-Tal Y, Wente SR, Cole CN: The Dbp5 cycle at the nuclear pore complex during mRNA export l: dbp5 mutants with defects in RNA binding and ATP hydrolysis define key steps for Nup159 and Gle1. Genes Dev 2011, 25(10):1052-1064.

5. Noble KN, Tran EJ, Alcazar-Roman AR, Hodge CA, Cole CN, Wente SR: The Dbp5 cycle at the nuclear pore complex during mRNA export II: nucleotide cycling and mRNP remodeling by $\mathrm{Dbp} 5$ are controlled by Nup159 and Gle1. Genes Dev 2011, 25(10):1065-1077.

6. Lund MK, Guthrie C: The DEAD-box protein Dbp5p is required to dissociate Mex67p from exported mRNPs at the nuclear rim. Mol Cell 2005, 20(4):645-651.

7. Tran EJ, Zhou Y, Corbett AH, Wente SR: The DEAD-box protein Dbp5 controls mRNA export by triggering specific RNA:protein remodeling events. Mol Cell 2007, 28(5):850-859.

8. Snay-Hodge CA, Colot HV, Goldstein AL, Cole CN: Dbp5p/Rat8p is a yeast nuclear pore-associated DEAD-box protein essential for RNA export. EMBO J 1998, 17(9):2663-2676.

9. Tseng SS, Weaver PL, Liu Y, Hitomi M, Tartakoff AM, Chang TH: Dbp5p, a cytosolic RNA helicase, is required for poly $(A)+$ RNA export. EMBO J 1998, 17(9):2651-2662.

10. Zhao J, Jin SB, Bjorkroth B, Wieslander L, Daneholt B: The mRNA export factor Dbp5 is associated with Balbiani ring mRNP from gene to cytoplasm. EMBO J 2002, 21(5):1177-1187.

11. Estruch F, Cole CN: An early function during transcription for the yeast mRNA export factor Dbp5p/Rat8p suggested by its genetic and physical interactions with transcription factor IIH components. Mol Biol Cell 2003, 14(4):1664-1676

12. Pandit $S$, Wang $D$, Fu XD: Functional integration of transcriptional and RNA processing machineries. Curr Opin Cell Biol 2008, 20(3):260-265.

13. Licatalosi DD, Darnell RB: RNA processing and its regulation: global insights into biological networks. Nat Rev Genet 2010, 11(1):75-87.

14. Rodriguez-Navarro $S$, Hurt E: Linking gene regulation to mRNA production and export. Curr Opin Cell Biol 2011, 23(3):302-309.

15. Rondon $A G$, Jimeno $S$, Aguilera $A$ : The interface between transcription and mRNP export: from THO to THSC/TREX-2. Biochim Biophys Acta 2010 1799(8):533-538

16. Rondon AG, Garcia-Rubio M, Gonzalez-Barrera S, Aguilera A: Molecular evidence for a positive role of Spt4 in transcription elongation. EMBO J 2003, 22(3):612-620.

17. Strasser K, Masuda S, Mason P, Pfannstiel J, Oppizzi M, Rodriguez-Navarro S, Rondon AG, Aguilera A, Struhl K, Reed $R$, et al: TREX is a conserved complex coupling transcription with messenger RNA export. Nature 2002, 417(6886):304-308

18. Strasser K, Hurt E: Yra1p, a conserved nuclear RNA-binding protein, interacts directly with Mex67p and is required for mRNA export. EMBO J 2000, 19(3):410-420.
19. Schmitt C, von Kobbe C, Bachi A, Pante N, Rodrigues JP, Boscheron C, Rigaut G, Wilm M, Seraphin B, Carmo-Fonseca M, et al: Dbp5, a DEAD-box protein required for mRNA export, is recruited to the cytoplasmic fibrils of nuclear pore complex via a conserved interaction with CAN/Nup159p. EMBO J 1999, 18(15):4332-4347.

20. Jensen TH, Boulay J, Rosbash M, Libri D: The DECD box putative ATPase Sub2p is an early mRNA export factor. Curr Biol 2001, 11(21):1711-1715.

21. Jensen TH, Boulay J, Olesen JR, Colin J, Weyler M, Libri D: Modulation of transcription affects mRNP quality. Mol Cell 2004, 16(2):235-244.

22. Mermelstein $F$, Yeung $K$, Cao J, Inostroza JA, Erdjument-Bromage $H_{\text {, }}$ Eagelson K, Landsman D, Levitt P, Tempst P, Reinberg D: Requirement of a corepressor for Dr1-mediated repression of transcription. Genes Dev 1996, 10(8):1033-1048.

23. Goppelt A, Meisterernst M: Characterization of the basal inhibitor of class II transcription NC2 from Saccharomyces cerevisiae. Nucleic Acids Res 1996, 24(22):4450-4455.

24. Peiro-Chova L, Estruch F: Specific Defects in Different Transcription Complexes Compensate for the Requirement of the Negative Cofactor 2 Repressor in Saccharomyces cerevisiae. Genetics 2007, 176(1):125-138.

25. Xie J, Collart M, Lemaire M, Stelzer G, Meisterernst M: A single point mutation in TFIIA suppresses NC2 requirement in vivo. EMBO J 2000 19(4):672-682

26. Ozer J, Lezina LE, Ewing J, Audi S, Lieberman PM: Association of transcription factor IIA with TATA binding protein is required for transcriptional activation of a subset of promoters and cell cycle progression in Saccharomyces cerevisiae. Mol Cell Biol 1998, 18(5):2559-2570.

27. Matsui P, DePaulo J, Buratowski S: An interaction between the Tfb1 and Ssl1 subunits of yeast TFIIH correlates with DNA repair activity. Nucleic Acids Res 1995, 23(5):767-772

28. Yoon H, Miller SP, Pabich EK, Donahue TF: SSL1, a suppressor of a HIS4 $5^{\prime}$-UTR stem-loop mutation, is essential for translation initiation and affects UV resistance in yeast. Genes Dev 1992, 6(12B):2463-2477.

29. Segref A, Sharma K, Doye V, Hellwig A, Huber J, Luhrmann R, Hurt E: Mex67p, a novel factor for nuclear mRNA export, binds to both poly(A) + RNA and nuclear pores. EMBO J 1997, 16(11):3256-3271.

30. Hilleren P, McCarthy T, Rosbash M, Parker R, Jensen TH: Quality control of mRNA 3/-end processing is linked to the nuclear exosome. Nature 2001 413(6855):538-542

31. Estruch F, Hodge CA, Rodriguez-Navarro S, Cole CN: Physical and genetic interactions link the yeast protein Zds1p with mRNA nuclear export. J Biol Chem 2005, 280(10):9691-9697.

32. Chavez $S$, Aguilera A: The yeast HPR1 gene has a functional role in transcriptional elongation that uncovers a novel source of genome instability. Genes Dev 1997, 11(24):3459-3470.

33. Hartzog GA, Wada T, Handa H, Winston F: Evidence that Spt4, Spt5, and Spt6 control transcription elongation by RNA polymerase II in Saccharomyces cerevisiae. Genes Dev 1998, 12(3):357-369.

34. Wittschieben $\mathrm{BO}$, Otero $\mathrm{G}$, de Bizemont $\mathrm{T}$, Fellows J, Erdjument-Bromage $\mathrm{H}$, Ohba R, Li Y, Allis CD, Tempst P, Svejstrup JQ: A novel histone acetyltransferase is an integral subunit of elongating RNA polymerase II holoenzyme. Mol Cell 1999, 4(1):123-128.

35. Kim HJ, Jeong SH, Heo JH, Jeong SJ, Kim ST, Youn HD, Han JW, Lee HW, Cho EJ: mRNA capping enzyme activity is coupled to an early transcription elongation. Mol Cell Biol 2004, 24(14):6184-6193.

36. Myers $L C$, Lacomis $L$, Erdjument-Bromage $H$, Tempst $P$ : The yeast capping enzyme represses RNA polymerase II transcription. Mol Cell 2002, 10 (4):883-894.

37. Schroeder SC, Zorio DA, Schwer B, Shuman S, Bentley D: A function of yeast mRNA cap methyltransferase, Abd1, in transcription by RNA polymerase II. Mol Cell 2004, 13(3):377-387.

38. Jensen TH, Patricio K, McCarthy T, Rosbash M: A block to mRNA nuclear export in S. cerevisiae leads to hyperadenylation of transcripts that accumulate at the site of transcription. Mol Cell 2001, 7(4):887-898.

39. Winston F, Dollard C, Ricupero-Hovasse SL: Construction of a set of convenient Saccharomyces cerevisiae strains that are isogenic to S288C. Yeast 1995, 11(1):53-55

40. Strasser K, Hurt E: Splicing factor Sub2p is required for nuclear mRNA export through its interaction with Yra1p. Nature 2001, 413(6856):648-652. 
41. Sikorski RS, Hieter P: A system of shuttle vectors and yeast host strains designed for efficient manipulation of DNA in Saccharomyces cerevisiae. Genetics 1989, 122(1):19-27.

42. Cole CN, Heath CV, Hodge CA, Hammell CM, Amberg DC: Analysis of RNA export. Methods Enzymol 2002, 351:568-587.

43. Forrester W, Stutz F, Rosbash M, Wickens M: Defects in mRNA 3'-end formation, transcription initiation, and mRNA transport associated with the yeast mutation prp20: possible coupling of mRNA processing and chromatin structure. Genes Dev 1992, 6(10):1914-1926.

doi:10.1186/1471-2156-13-80

Cite this article as: Estruch et al:: Insights into mRNP biogenesis

provided by new genetic interactions among export and transcription

factors. BMC Genetics 2012 13:80.

\section{Submit your next manuscript to BioMed Central and take full advantage of:}

- Convenient online submission

- Thorough peer review

- No space constraints or color figure charges

- Immediate publication on acceptance

- Inclusion in PubMed, CAS, Scopus and Google Scholar

- Research which is freely available for redistribution 